\title{
Natural Products for Cancer Prevention and Therapy: Progress, Pitfalls and Promise ${ }^{\dagger}$
}

\author{
Anupam Bishayee \\ Department of Pharmaceutical Sciences, College of Pharmacy, Larkin University, Miami, FL 33169, USA; \\ abishayee@ularkin.org or abishayee@gmail.com; Tel.: +1-305-760-7511 \\ + Presented at the 2nd International Conference on Natural Products for Cancer Prevention and Therapy, \\ Kayseri, Turkey, 8-11 November 2017.
}

Published: 15 November 2017

\begin{abstract}
Natural products represent a valuable resource for discovery and development of anticancer drugs. About $80 \%$ of drugs approved by the United States Food and Drug Administration during the last three decades for cancer therapy are either natural products per se or are based on, or mimicked natural products. Various extracts, fractions, mixtures and pure compounds from dietary sources, medicinal plants and marine organisms have been found to exhibit potent antiproliferative and cytotoxic activities against numerous cancer cell lines. These agents have also been tested for cancer preventive and therapeutic efficacies using preclinical animal cancer models that resemble human disease. Selected natural products have also entered into clinical studies. The cancer preventive and anticancer attributes of various natural products can be explained by multiple cellular and molecular mechanisms, including scavenging of free radicals, detoxification of carcinogens, DNA repair, alteration of cell cycles, programmed cell death (apoptosis), autophagy, immune surveillance, anti-inflammatory, anti-angiogenic, anti-invasive and antimetastatic activities as well as their ability to modulate a plethora of dysregulated oncogenic signaling pathways. This presentation aims to highlight studies on cancer preventive and therapeutic attributes of various naturally occurring agents and underlying mechanisms of action, with special emphasis on results reported from our laboratory. Current limitations, challenges, and future directions of research for successful cancer drug development based on natural products will also be discussed.
\end{abstract}

Keywords: cancer; chemoprevention; therapy; resveratrol; anthocyanins; triterpenoids

Conflicts of Interest: The authors declare no conflict of interest.

(C) 2017 by the authors. Licensee MDPI, Basel, Switzerland. This article is an open access article distributed under the terms and conditions of the Creative Commons Attribution (CC BY) license (http://creativecommons.org/licenses/by/4.0/). 